\title{
Notes on Noetherian Semigroup Rings
}

\author{
Ryûki MatsudA*
}

Let $A$ be a commutative ring with identity and let $S$ be a torsion-free cancellative commutative semigroup with identity. We write the semigroup operation on $S$ as addition and assume that $S \supsetneq\{0\}$. We consider the semigroup ring $A[X ; S]$ of $S$ over $A$. In [12] we determined conditions under which $A[X ; S]$ is a Noetherian ring. We concern with $A[X ; S]$ further as a Noetherian ring in this paper. Throughout this paper $A$ denotes a commutative ring with identity. $S$ denotes the above mentioned semigroup. The smallest group containing $S$ is called quotient group of $S$ and is denoted by $q(S)$. $G$ denotes the quotient group of $S$.

\section{1.}

At first we list some Noetherian properties of a ring.

DeFinItion 1. The followings are some Noetherian properties of a ring and references definitions of which are due to:

Cohen-Macaulay ring $[13,(16 . \mathrm{E})]$;

universally catenary $[13$, p. 84$]$

excellent ring [13, (34. A)];

Nagata ring $[13,(31 . A)]$;

G-ring [13, (33. A)];

integrally Noetherian ring [2, chap. $5, \S 3$, exerc. 6$)]$;

$J-0$ ring [13, (32. B)];

$J-2$ ring $[13,(32 . \mathrm{B})]$.

THEOREM 2([8], [9], [10]). Let $\pi$ be one of properties of a ring of Definition 1. If $S$ is a group, $A[X ; S]$ is a ring with property $\pi$ if and only if $A$ is a ring with property $\pi$ and $S$ is a finitely generated group.

\section{THEOREM 3.}

(1) (a special case of $[12$, Appendix $]$ ) $A[X ; S]$ is a Noetherian ring if and only if $A$ is a Noetherian ring and $S$ is a finitely generated semigroup;

(2) $A[X ; S]$ is an $r$-Noetherian ring if and only if $A[X ; S]$ is a Noetherian ring. Using the results of Connell (Canad. J. Math., 15(1963)) and of [9], the proof

Received February 28, 1983, Research partially supported by the Grant-in-Aid for Scientific Research C-57540005 (1982) from the Ministry of Education.

- Department of Mathematics, Ibaraki University, Mito, Ibaraki 310, Japan. 
of (2) is similar with that of [12, Appendix $]$. We omit it.

Here, let $Z_{0}$ be the set of nonnegative integers. If there exists a finite subset $\left\{s_{1}, \ldots, s_{n}\right\}$ of $S$ such that $S=Z_{0} s_{1}+\cdots+Z_{0} s_{n}, S$ is said to be a finitely generated semigroup. And, if each ideal of a ring $A$ containing a nonzerodivisor is a finitely generated ideal, $A$ is said to be an $r$-Noetherian ring ([7]).

As a corollary to Theorem 3, we can generalize a part of Theorem 2 for a semigroup;

THEOREM 4. $A[X ; S]$ is a ring with the respective property of universally catenary, excellent, Nagata, G-ring, integrally Noetherian and $J-2$, if and only if $A$ is a ring with the respective property and $S$ is a finitely generated semigroup.

Proof. Assume that $A[X ; S]$ is a universally catenary ring. $A[X ; G]$ is a quotient ring of $A[X ; S]$. Therefore $A[X ; G]$ is a universally catenary. By Theorem $2, A$ is universally catenary ring. Since $A[X ; S]$ is a Noetherian ring by definition, $S$ is a finitely generated semigroup by Theorem 3 . Conversely assume that $A$ is a universally catenary ring and $S$ is a finitely generated semigroup. Then $A[X ; S]$ is a finitely generated algebra over $A$. Therefore $A[X ; S]$ is a universally catenary ring. Proofs for the respective property of excellent, Nagata, $G$-ring and integrally Noetherian are similar with that for universally catenary. Let $A$ be a ring with the property $J-2$ and let $S$ be a finitely generated semigroup. A finitely generated algebra of $A[X ; S]$ is a finitely generated algebra over $A$. It follows that $A[X ; S]$ is a ring with the property $J-2$. Conversely assume that $A[X ; S]$ is a ring with the property of $J-2$. Let $P$ be a prime ideal of $A$. We set $\varphi^{-1}(P)=\mathfrak{p}$ in the following Lemma 5 . Since $A / P=A[X ; S] / \mathfrak{p}, A$ is a ring with the property of $J-2$ by [13, Theorem 73].

LEMMA 5. There exists a canonical ring homomorphism $\varphi$ of $A[X ; S]$ onto $A$.

Proof. There exists a canonical ring homomorphism $\psi$ of $A[X ; G]$ onto $A$. Then the restriction $\varphi$ of $\psi$ to $A[X ; S]$ is a desired homomorphism.

Let $D$ be an integral domain. We note that Bouvier [3] asks to determine conditions under which $D[X ; S]$ is a catenary ring and also asks to determine conditions under which $D[X ; S]$ is a Cohen-Macaulay ring.

THEOREM 6. $A[X ; S]$ has property $J-0$ if and only if $A$ has property $J-0$ and $S$ is a finitely generated semigroup.

Proof. Sufficiency. Since $G$ is a finitely generated group, $A[X ; G]$ is a ring with property $J-0$ by Theorem 2 . Theorefore there exists an element $f \in A[X ; G]$ such that $\emptyset \neq D^{\prime}(f) \subset \operatorname{Reg}(A[X ; G])$. Here $D^{\prime}(f)$ is the set of prime ideals $P$ of $A[X ; G]$ such that $f \bar{\in} P$ and $\operatorname{Reg}(A[X ; G])$ is the set of prime ideals $P$ of $A[X ; G]$ 
such that $A[X ; G]_{P}$ is a regular local ring. Set $S=\sum_{1}^{n} Z_{0} s_{i}$. Then the set $D\left(f X^{s_{1}} \ldots\right.$ $\left.X^{s_{n}}\right)$ of prime ideals $P$ of $A[X ; S]$ such that $P \bar{\nexists} f X^{s_{1}} \ldots X^{s_{n}}$ is not an empty set.

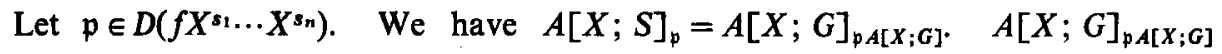
is a regular local ring, since $\mathfrak{p} A[X ; G] \bar{\exists} f$. We have proved that $A[X ; S]$ is a ring with the property $J-0$. Necessity. There exists $f \in A[X ; S]$ such that $\emptyset \neq D(f) \subset \operatorname{Reg}(A[X ; S])$. Set $f=a_{1} X^{s_{1}}+\cdots+a_{n} X^{s_{n}}$, where $0 \neq a_{i} \in A$ and $s_{1}<$ $s_{2}<\cdots<s_{n}(G=q(S)$ becomes a totally ordered group). Since $\emptyset \neq D(f)$, there exists $k$ such that $a_{k}$ is not a nilpotent element. Then the set $D_{0}(a)=\{P$; prime ideal of $A$ such that $P \bar{\exists} a\}$ is not an empty set. Let $P \in D_{0}(a)$. Then $P A[X ; S] \in D(f) \subset$

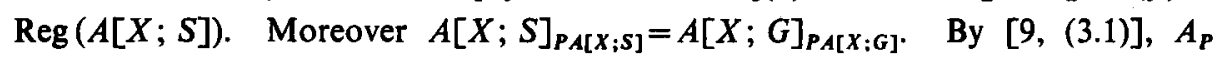
is a regular local ring. Therefore $A$ has property $J-0$.

2.

Proposition 1. If $A[X ; S]$ is a Cohen-Macaulay ring, then $A$ is a CohenMacaulay ring and $S$ is a finitely generated semigroup.

Proof. It follows that $S$ is a finitely generated semigroup by Theorem 3 of section 1 and that $A[X ; G]$ is a Cohen-Macaulay ring. Then $A$ is a CohenMacaulay ring by Theorem 2 of section 1 .

LeMmA $2([10,(6.5)])$. If $A$ is a Noetherian ring, then we have $\operatorname{dim} A[X ; S]=$ $\operatorname{dim} A[X ; G]=\operatorname{dim} A+$ t. f. r. $(G)$.

Here t.f.r. $(G)$ is the torsion-free rank of $G$.

LEMMA 3.

(1) (e.g. [5, (31.16]). An integral domain which is a finitely generated algebra over a field satisfies the first chain condition (f.c.c.).

(2) ([1, chap. II, §1, Prop. 2]). Let a be a subset of a ring $A$ which is both additively and multiplicatively closed. Let $P_{1}, \ldots, P_{n}$ be a finite number of prime ideals of $A$ so that $a$ is contained in the set-theoretical union $P_{1} \cup \cdots \cup P_{n}$. Then $\mathfrak{a}$ is contained in some $P_{i}$.

(3) (e.g. [13, (16.D)]). Let $A$ be a Noetherian ring. Then $A$ is a CohenMacaulay ring if and only if the unmixed theorem holds in $A$.

(4) (e.g. [13, Theorem 31]). Let $A$ be a Cohen-Macaulay ring and let $\left\{X_{1}, \ldots\right.$, $\left.X_{n}\right\}$ be a system of indeterminates over $A$. Then a quotient ring of $A\left[X_{1}, \ldots, X_{n}\right]$ is a Cohen-Macaulay ring.

Lemma 4. Let $k^{\prime}$ be a field and let $k$ be a subfield of $k^{\prime}$. Let $M^{\prime}$ be a prime ideal of $k^{\prime}[X ; S]$ and let $M$ be a prime ideal of $k[X ; S]$ such that $M^{\prime} \cap k[X ; S]=$ $M$. Let $m_{1}, \ldots, m_{s}$ be a finite set of elements of $M$. Then $\left\{m_{1}, \ldots, m_{s}\right\}$ is a regular sequence for $k^{\prime}[X ; S]_{M^{\prime}}$ if and only if it is a regular sequence for $k[X ; S]_{M}$. 
ProOF. Let $\left\{u_{\lambda} ; \lambda \in \Lambda\right\}$ be a linear basis of $k^{\prime}$ over $k$. Then $k^{\prime}[X ; S]$ is a free module over $k[X ; S]$ and $\left\{u_{\lambda} ; \lambda \in \Lambda\right\}$ is a free basis of $k^{\prime}[X ; S]$ over $k[X ; S]$. Assume $\left\{m_{1}, \ldots, m_{s}\right\}$ is a regular sequence for $k^{\prime}[X ; S]_{M^{\prime}}$. Let $1 \leq l \leq s$ and let $\xi \in k[X ; S]_{M}$. Assume $\xi m_{l} \in\left(m_{1}, \ldots, m_{l-1}\right) k[X ; S]_{M}$. There exist $f, g, f_{1}, \ldots, f_{l-1}$ $\in k[X ; S]$ such that $g \bar{\in} M, \xi=f / g$ and $f m_{l}=\sum_{1}^{l-1} m_{i} f_{i}$. We have $f=\sum_{1}^{l-1} m_{i} F_{i} / G$ for $F_{1}, \ldots, F_{l-1}, G \in k^{\prime}[X ; S]$ such that $G \bar{\in} M^{\prime}$. Express $F_{i}=\sum f_{i \lambda} u_{\lambda}$ and $G=\sum g_{\lambda} u_{\lambda}$ where $f_{i \lambda}, g_{\lambda} \in k[X ; S]$. Since $G \bar{\in} M^{\prime}$, there exists $\lambda_{0} \in \Lambda$ such that $g_{\lambda_{0}} \bar{\in} M$. We have $f g_{\lambda 0}=\sum_{1}^{l-1} m_{i} f_{i \lambda_{0}}$. Since $\xi=\sum m_{i}\left(f_{i \lambda_{0}}\right) /\left(g g_{\lambda_{0}}\right)$, we have $\xi \in \sum_{1}^{l-1} m_{i} k[X ; S]_{M}$. Therefore $\left\{m_{1}, \ldots, m_{s}\right\}$ is a regular sequence for $k[X ; S]_{M}$. Conversely assume that $\left\{m_{1}, \ldots, m_{s}\right\}$ is a regular sequence for $k[X ; S]_{M}$. Let again $1 \leq l \leq s$ and let $\eta \in$ $k^{\prime}[X ; S]_{M^{\prime}}$ such that $\eta m_{l} \in\left(m_{1}, \ldots, m_{l-1}\right) k^{\prime}[X ; S]_{M}{ }^{\prime}$. There exist $F, G, F_{1}, \ldots$, $F_{l-1} \in k^{\prime}[X ; S]$ such that $G \bar{\in} M^{\prime}, \eta=F / G$ and $F m_{l}=\sum_{1}^{l-1} m_{i} F_{i}$. Express $F=$ $\sum f_{\lambda} u_{\lambda}$ and $F_{i}=\sum f_{i \lambda} u_{\lambda}$ for $f_{\lambda}, f_{i \lambda} \in k[X ; S]$. We have $f_{\lambda} m_{l}=\sum_{i}^{l-1} f_{i \lambda} m_{i}$. It follows $f_{\lambda} \in\left(m_{1}, \ldots, m_{l-1}\right) k[X ; S]_{M}$. It follows $\eta \in\left(m_{1}, \ldots, m_{l-1}\right) k^{\prime}[X ; S]_{M^{\prime}}$. Therefore $\left\{m_{1}, \ldots, m_{s}\right\}$ is a regular sequence for $k^{\prime}[X ; S]_{M^{\prime}}$.

Proposition 5. Let $k^{\prime}$ be an algebraic extension field of a field $k$. Then if $k[X ; S]$ is a Cohen-Macaulay ring, $k^{\prime}[X ; S]$ is a Cohen-Macaulay ring.

Proof. Let $M^{\prime}$ be a maximal ideal of $k^{\prime}[X ; S]$. Set $M^{\prime} \cap k[X ; S]=M$. Since $k^{\prime}[X ; S]$ is integral over $k[X ; S], M$ is a maximal ideal of $k[X ; S]$. We set t. f. r. $(G)=d$. We have $\operatorname{dim} k^{\prime}[X ; S]=\operatorname{dim} k[X ; S]=d$ by Lemma 2 . We have $h t\left(M^{\prime}\right)=h t(M)=d$ by Lemma $3(1)$ (' $h t$ ' means the height). There exists a finite set $m_{1}, \ldots, m_{d}$ of $d$ elements of $M$ such that $\left\{m_{1}, \ldots, m_{d}\right\}$ is a regular sequence for $k[X ; S]_{M}$. $\quad\left\{m_{1}, \ldots, m_{d}\right\}$ is a regular sequence for $k^{\prime}[X ; S]_{M^{\prime}}$ by Lemma 4 . Since $k^{\prime}[X ; S]$ is a Noetherian ring by Theorem 3 of section $1, k^{\prime}[X ; S]$ is a CohenMacaulay ring.

Proposition 6. Let $k^{\prime}$ be a purely-transcendental extension field of $k$. Then, if $k[X ; S]$ is a Cohen-Macaulay ring $k^{\prime}[X ; S]$ is a Cohen-Macaulay ring.

Proof. Let $\left\{v_{\sigma} ; \sigma \in \Sigma\right\}$ be a purely-transcendence basis of $k^{\prime}$ over $k$. Let $M^{\prime}$ be a maximal ideal of $k^{\prime}[X ; S]$. We have $h t\left(M^{\prime}\right)=d(=$ t. f. r. $(G))$. Let $v_{1}, \ldots$, $v_{n}$ be a finite number of elements of $\left\{v_{\sigma} ; \sigma \in \Sigma\right\}$. We set $k_{0}=k\left(v_{1}, \ldots, v_{n}\right)$ and $k_{0}[X ; S] \cap M^{\prime}=M_{0}$. We can choose such elements $v_{1}, \ldots, v_{n}$ that $h t\left(M_{0}\right)=d$. Then $M_{0}$ is a maximal ideal of $k_{0}[X ; S]$. Since $k_{0}[X ; S]$ is a quotient ring of $(k[X ; S])\left[v_{1}, \ldots, v_{n}\right], k_{0}[X ; S]$ is a Cohen-Macaulay ring by Lemma $3(4)$. There exists a finite number of elements $m_{1}, \ldots, m_{d}$ of $M_{0}$ such that $\left\{m_{1}, \ldots, m_{d}\right\}$ is a regular sequence for $k_{0}[X ; S]_{M_{0}}$. Since $\left\{m_{1}, \ldots, m_{d}\right\}$ is a regular sequence for $k^{\prime}[X ; S]_{M}$, by Lemma $4, k^{\prime}[X ; S]_{M}$ is a Cohen-Macaulay ring. 
Lemma 7. Let $k^{\prime}$ be an extension field of $k$. Let $M$ be a maximal ideal of $k[X ; S]$ and let $M^{\prime}$ be a maximal ideal of $k^{\prime}[X ; S]$ such that $M^{\prime} \cap k[X ; S]=M$. Then $M^{\prime}$ is a minimal prime ideal of $M k^{\prime}[X ; S]$.

Proof. Let $\left\{v_{\sigma} ; \sigma \in \Sigma\right\}$ be a transcendence basis of $k^{\prime}$ over $k$. Set $k_{0}=$ $k\left(v_{\sigma} ; \sigma \in \Sigma\right)$ and set $M^{\prime} \cap k_{0}[X ; S]=M_{0} . \quad k_{0}[X ; S]$ is a quotient ring of a polynomial ring $(k[X ; S])\left[v_{\sigma} ; \sigma \in \Sigma\right]$ over $k[X ; S]$. Hence $M k_{0}[X ; S]$ is a prime ideal of $k_{0}[X ; S]$ of $h t \geq d$ (=t.f.r. $(G)$ ), and hence $M_{0}=M k_{0}[X ; S]$. Since $k^{\prime}[X ; S]$ is integral over $k_{0}[X ; S]$, each prime ideal of $k^{\prime}[X ; S]$ lying over $M_{0}$ is a maximal ideal of $k^{\prime}[X ; S]$. Therefore $M^{\prime}$ is a minimal prime ideal of $M k^{\prime}[X$; S].

Proposition 8. Let $k^{\prime}$ be an extension field of a field $k$. Then if $k^{\prime}[X ; S]$ is a Cohen-Macaulay ring, $k[X ; S]$ is a Cohen-Macaulay ring.

Proof. Let $M$ be a maximal ideal of $k[X ; S]$. Since $k^{\prime}[X ; S]$ is a free module over $k[X ; S]$, there exists a maximal ideal $M^{\prime}$ such that $M^{\prime} \cap k[X ; S]=$ $M$. Let $\left\{m_{1}, \ldots, m_{s}\right\}$ be a maximal regular sequence for $k[X ; S]_{M}$. We may assume $m_{i} \in M$ for $1 \leq i \leq s$. Suppose $s<d(=$ t.f.r. $(G)) . \quad\left\{m_{1}, \ldots, m_{s}\right\}$ is a regular sequence for $k^{\prime}[X ; S]_{M}$, by Lemma 4 . Let $\mathfrak{P}_{1}, \ldots, \mathfrak{P}_{k}$ be the prime ideals belonging to $\left(m_{1}, \ldots, m_{s}\right) k^{\prime}[X ; S]_{M^{\prime}}$. We have $h t\left(\mathfrak{P}_{i}\right)=s$ for $1 \leq i \leq s$ by Lemma 3(3). By Lemma $7, M$ is not contained in any $\mathfrak{P}_{i}$. It follows $M \odot \mathfrak{P}_{1} \cup \cdots \cup \mathfrak{P}_{k}$ by Lemma 3(2). We take an element $m \in M$ such that $m \bar{E} \mathfrak{P}_{1} \cup \cdots \cup \mathfrak{P}_{k}$. Then $\left\{m_{1}, \ldots, m_{s}, m\right\}$ is a regular sequence for $k^{\prime}[X ; S]_{M^{\prime}} . \quad\left\{m_{1}, \ldots, m_{s}, m\right\}$ is a regular sequence for $k[X ; S]_{M}$ by Lemma 4 , which is a contradiction. Therefore $k[X ; S]_{M}$ is a Cohen-Macaulay ring.

Proposition 5, 6 and 8 implies the following result:

THEOREM 9.

(1) Let $k^{\prime}$ be an extension field of $k$. Then $k^{\prime}[X ; S]$ is a Cohen-Macaulay ring if and only if $k[X ; S]$ is a Cohen-Macaulay ring.

(2) Let $k$ be a field and let $k_{0}$ be a prime field of $k$. Then $k[X ; S]$ is a CohenMacaulay ring if and only if $k_{0}[X ; S]$ is a Cohen-Macaulay ring.

3.

Let $n$ be a fixed positive integer throughout this section. If each finitely generated ideal of a ring $A$ is generated by $n$ elements of $A, A$ is said to have $n$ generator property $([5$, p. 320$])$.

Proposinon 1([9, (4.16)]). Let $D$ be a domain and assume that $S$ is a group. Then $D[X ; S]$ has n-generator property if and only if $D$ is a field and $S$ is isomorphic into $Q$. 
We denote the set of nonnegative rational numbers by $Q_{0}$. Let $S$ be a finitely generated (additive) subsemigroup of $Q_{0}$. We have $q(S)=Z e$ for $e>0$. Then the smallest natural number contained in $(1 / e) S$ we will call the order of $S$. Next, if each ideal of a ring $A$ is generated by $n$ elements $A$ is said to have rank $n([4])$. We determined in $[11$, section 5] conditions under which $A[X ; S]$ has rank $n$. Especially we have

Lemma $2([11,(5.6)])$. Let $D$ be a domain. Then $D[X ; S]$ has rank $n$ if and only if $D$ is a field and $S$ is either isomorphic with $Z$ or isomorphic with a subsemigroup of $Z_{0}$ containing a natural number $m \leq n$.

THEOREM 3. Let $D$ be a domain. Then $D[X ; S]$ has n-generator property if and only if $D$ is a field and $S$ is either isomorphic with a subgroup of $Q$ or isomorphic with a subsemigroup $T$ of $Q_{0}$ such that if $T^{\prime}$ is a finitely generated subsemigroup of $T, T^{\prime}$ is contained in a finitely generated subsemigroup $T^{\prime \prime}$ of $T$ of order $\leq n$.

Proof. Sufficiency. We may assume that $S$ is a subsemigroup of $Q_{0}$ by Proposition 1. Let $a=\left(f_{1}, \ldots, f_{m}\right)$ be a finitely generated ideal of $D[X ; S]$. There exists a finite subset $\left\{s_{1}, \ldots, s_{l}\right\}$ of $S$ such that $f_{i} \in D\left[X ; \sum_{i}^{l} Z_{0} s_{i}\right]$ for $1 \leq i \leq l$. $\sum_{1}^{l} Z_{0} s_{i}$ is contained in a finitely generated subsemigroup $T^{\prime}$ of $S$ of order $\leq n$. By Lemma 2, there exists $n$ elements $g_{1}, \ldots, g_{n}$ in $D\left[X ; T^{\prime}\right]$ such that $\left(f_{1}, \ldots, f_{m}\right) D[X$; $\left.T^{\prime}\right]=\left(g_{1}, \ldots, g_{n}\right) D\left[X ; T^{\prime}\right]$. Then $a$ is an ideal of $D[X ; S]$ generated by $n$ elements. Therefore $D[X ; S]$ has $n$-generator property. Necessity. It follows that $D[X ; G]$ has $n$-generator property. Therefore $D$ is a field and $G$ is isomorphic into $Q$ by Proposition 1. We may assume that $S$ is a subsemigroup of $Q_{0}$. If our assertion is false, there exists a finitely generated subsemigroup $T$ of $S$ such that the order $m$ of $T$ is greater than $n$ and such that each finitely generated subsemigroup of $S$ containing $T$ has order $\geq m$. We may assume $q(T)=Z$. There exists a natural number $h$ such that $\{h, h+1, h+2, \ldots\} \subset T . \quad$ By Lemma $4,\left(X^{h}, X^{h+1}, \ldots, X^{h+m-1}\right)$ is not generated by $n$ elements, which is a contradiction.

Lemma 4. Let $k$ be a field and $S$ be a subsemigroup of $Q_{0}$. Assume $S$ has a finitely generated subsemigroup $T$ such that order of $T=m, q(T)=Z$ and each finitely generated subsemigroup of $S$ containing which has order $\geq m$. Assume $\{h, h+1, h+2, \ldots\} \subset T$.

(1) Let $k \in Z, 1 \leq k \leq m-1$. Let $g_{1}, \ldots, g_{k} \in\left(X^{h}, X^{h+1}, \ldots, X^{h+m-1}\right)$ such that $o\left(g_{1}\right)=h, o\left(g_{2}\right)=h+1, \ldots, o\left(g_{k}\right)=h+k-1$, and let $\varphi_{1}, \ldots, \varphi_{k} \in k[X ; S]$. Then we have $o\left(g_{1} \varphi_{1}+\cdots+g_{k} \varphi_{k}\right) \bar{\epsilon}\{h+k, h+k+1, \ldots, h+m-1\}$.

(2) $\left(X^{h}, X^{h+1}, \ldots, X^{h+m-1}\right)$ is not generated by $m-1$ elements of $k[X ; S]$.

Proof. We note that $1,2, \ldots, m-1 \bar{\epsilon} S$. (1). We show (1) by induction 
on $k$. Suppose that (1) is true for $k-1$. Suppose $o\left(g_{1} \varphi_{1}+\cdots+g_{k} \varphi_{k}\right)=\omega \epsilon$ $\{h+k, h+k+1, \ldots, h+m-1\}$. By induction we may assume that each $\varphi_{i}$ is either 0 or $o\left(\varphi_{i}\right)>0$. $\omega$ appears as one of the powers of $g_{i} \varphi_{i}$ for some $i$. Each power in $g_{i}$ is of the form $h+p+s$ where $Z \ni p, 0 \leq p \leq m-1, s \in S$. We have $h+p+s+s^{\prime}=\omega$ for $0<s^{\prime} \in S$. It follows that $S \ni s+s^{\prime}>0, Z \ni s+s^{\prime} \leq m-1$, which is a contradiction. (2). Suppose that $\left(X^{h}, X^{h+1}, \ldots, X^{h+m-1}\right)$ is generated by $n$ elements $f_{1}, \ldots, f_{n}$, where $n<m$. We take the minimal number $n$ so that $\left(X^{h}, \ldots, X^{n+m-1}\right)$ is generated by $n$ elements. We set $o\left(f_{i}\right)=d_{i}, 1 \leq i \leq n$. We may assume that $h=d_{1}<d_{2}<\cdots<d_{n}$ and the coefficient of $X^{d_{i}}$ in $f_{i}$ is 1 for $1 \leq i \leq n$. Suppose $h+1<d_{2}$. Since $X^{h+1} \in\left(f_{1}, \ldots, f_{n}\right)$, we have $o\left(f_{1} \varphi_{1}\right)=h+1$ for some $\varphi_{1} \in k[X ; S]$, which contradicts to (1). Hence $h<d_{2} \leq h+1$. Suppose $h<d_{2}<$ $h+1$. Since $f_{2} \in\left(X^{h}, X^{h+1}, \ldots, X^{h+m-1}\right)$, it follows $d_{2}-h \in S$. Replace $f_{2}$ by $f_{2}-$ $f_{1} X^{d_{2}-h}$. We note that there exists a discrete subgroup of $Q$ containing $Z$ and all powers of $f_{1}, \ldots, f_{n}$. Thus we may assume $d_{2}=h+1$. Suppose $h+2<d_{3}$. Since $X^{h+2} \in\left(f_{1}, \ldots, f_{n}\right)$ we have $o\left(f_{1} \varphi_{1}+f_{2} \varphi_{2}\right)=h+2$ for $\varphi_{1}, \varphi_{2} \in k[X ; S]$, which contradicts to (1). Hence $h+1<d_{2} \leq h+2$. Suppose $h+1<d_{2}<h+2$. Since $f_{3} \in\left(X^{h}, X^{h+1}, \ldots, X^{h+m-1}\right)$, we have $d_{3}-h$ or $d_{3}-h-1 \in S$. Replace $f_{3}$ by $f_{3}-$ $f_{1} X^{d_{3}-h}$ or $f_{3}-f_{2} X^{d_{3}-h-1}$. Thus we may assume $d_{3}=h+2$. We continue the process. Thus we may assume $o\left(f_{i}\right)=h+i-1$ for each $i$. Then $X^{h+m-1} \bar{E}\left(f_{1}\right.$, $\ldots, f_{n}$ ) by $(1)$; a contradiction.

Lemma $5([6$, Cor. 3.1]). Let $S$ be a group. Then $A[X ; S]$ is a Bezout ring (i.e. a ring with 1-generator property) if and only if $A$ is a von Neumann regular ring and $S$ is isomorphic into $Q$.

LeMma 6. Let $A$ be a von Neumann regular ring. If $S$ is a subsemigroup of $Z_{0}$ containing $n$, then $A[X ; S]$ has n-generator property.

Proor. Let $\mathfrak{a}=\left(f_{1}, \ldots, f_{n+1}\right)$ be an ideal of $A[X ; S]$ generated by $n+1$ elements. Set $d_{i}=\operatorname{deg} f_{i}$ for $1 \leq i \leq n+1$. We show that $a$ is generated by $n$ elements by the induction on $\sum_{1}^{n+1} d_{i}$. There exist $k \neq l$ such that $d_{l}>d_{k}$ and $d_{l} \equiv$ $d_{k} \bmod (n)$. Set $d_{l}-d_{k}=n q$ and $f_{k}=a_{0}+\cdots+t X^{d_{k}}$. There exists an idempotent $e \in A$ such that $t A=e A$. Set $1-e=e^{\prime}, e A=A_{1}$ and $e^{\prime} A=A_{2}$. We have $A[X$; $S]=A_{1}[X ; S] \oplus A_{2}[X ; S]$ and $\mathfrak{a}=\left(e f_{1}, \ldots, e f_{n+1}\right) A_{1}[X ; S] \oplus\left(e^{\prime} f_{1}, \ldots, e^{\prime} f_{n+1}\right) A_{2}[X$; $S]$. Since $\operatorname{deg} e^{\prime} f_{k}<d_{k},\left(e^{\prime} f_{1}, \ldots, e^{\prime} f_{n+1}\right) A_{2}[X ; S]$ is generated by $n$ elements of $A_{2}[X ; S]$. Since et is a unit of $A_{1}$, there exists $b \in A_{1}$ such that $\operatorname{deg}\left(e f_{l}-b e f_{k} X^{n q}\right)$ $<d_{l}$. Since $\left(e f_{1}, \ldots, e f_{n+1}\right) A_{1}[X ; S]=\left(e f_{1}, \ldots, e f_{k}, \ldots, e f_{l}-b e f_{k} X^{n q}, \ldots, e f_{n+1}\right)$ $A_{1}[X ; S],\left(e f_{1}, \ldots, e f_{n+1}\right) A_{1}[X ; S]$ is generated by $n$ elements of $A_{1}[X ; S]$. Therefore $\mathfrak{a}$ is generated by $n$ elements.

Theorem 7. Assume that $A$ has the nilradical (0). Then $A[X ; S]$ has $n$-generator property if and only if $A$ is a von Neumann regular ring and $S$ is 
either isomorphic with a subgroup of $Q$ or isomorphic with a subsemigroup $T$ of $Q_{0}$ such that if $T^{\prime}$ is a finitely generated subsemigroup of $T, T^{\prime}$ is contained in a finitely generated subsemigroup $T^{\prime \prime}$ of $T$ of order $\leq n$.

Proof. The necessity. Let $P$ be a prime ideal of $A . \quad(A / P)[X ; S]$ has $n$ generator property. By Theorem $3, A / P$ is a field and $S$ is a desired semigroup. Since $A$ has dimension 0 and nilradical (0), $A$ is a von Neumann regular ring. The sufficiency. By Lemma 5 , we may assume that $S$ is a subsemigroup of $Q_{0}$. Let $\mathfrak{a}=\left(f_{1}, \ldots, f_{n+1}\right)$ be an ideal of $A[X ; S]$ generated by $n+1$ elements. There exists a finitely generated subsemigroup $T^{\prime}$ of $S$ of order $\leq n$ such that $f_{i} \in A\left[X ; T^{\prime}\right]$ for $1 \leq i \leq n+1$. By Lemma $6,\left(f_{1}, \ldots, f_{n+1}\right) A\left[X ; T^{\prime}\right]$ is generated by $n$ elements of $A\left[X ; T^{\prime}\right]$. Therefore $\mathfrak{a}$ is generated by $n$ elements of $A[X ; S]$.

\section{References}

[1] N. Bourbaki, Algèbre commutative, chap. 1, 2, Hermann, Paris, 1961.

[2] N. Bourbaki, Algèbre commutative, chap. 5, 6, Hermann, Paris, 1964.

[3] A. Bouvier, Anneaux de Krull graduès, Univ. Claude-Bernard, 1981.

[4] I. Cohen, Commutative rings with restricted minimum condition, Duke Math. Journ. 17 (1950), 27-42.

[5] R. Gilmer, Multiplicative ideal theory, Marcel Dekker, New York, 1972.

[6] R. Gilmer and T. Parker, Semigroup rings as Prüfer rings, Duke Math. Journ. 41 (1974), 219-230.

[7] R. Kennedy, A generalization of Krull domains to rings with zero divisors, Thesis, Univ. Missouri-Kansas (1973).

[8] R. Matsuda, Infinite group rings II, Bull. Fac. Sci., Ibaraki Univ. 8 (1976), 43-66.

[9] R. Matsuda, Torsion-free abelian group rings III, Bull. Fac. Sci., Ibaraki Univ. 9 (1977), $1-49$.

[10] R. Matsuda, Torsion-free abelian semigroup rings IV, Bull. Fac. Sci., Ibaraki Univ. 10 (1978), 1-27.

[11] R. Matsuda, Torsion-free abelian semigroup rings V, Bull. Fac. Sci., Ibaraki Univ. 11 (1979), 1-37.

[12] R. Matsuda, On Anderson-Anderson problem, preprint.

[13] H. Matsumura, Commutative algebra, Benjamin, New York, 1970. 